\title{
TREATMENT OF OPEN FRACTURES
}

\author{
By F. D. SANER, F.R.C.S.(Eng.) \\ (Surgeon, Royal Northern Hospital)
}

While disease has varied in character with differing civilisations and the circumstances of living, injuries have varied only in extent and the methods of infliction; the problem of their treatment has remained the same. The treatment of an open wound involving injury to bone -surely one of the most primitive of injuries-always has and always will include treatment of the wound, of the part injured, and of the person; the essential demands of treatment have never altered, but only during the last 25 to 30 years has it been possible to organise these on an appropriate basis. A very brief review of the history of treatment, confined to this period, is not only instructive but imperative to a proper perspective of the subject; it covers the brief interval since the days when amputation, immediate or remote, was the usual ending of an open fracture, and during which certain principles of treatment have been more surely recognised and insisted on as a basis of treatment. Within the framework of these principles there are variations of opinion on the actual practice, on the details of technique, but such differences may be regarded as competitive in an effort to fulfil more adequately the requirements of the principle. For example, the different methods of splinting are matters of technical detail, the principle involved is immobilisation of a part injured; again excision of a recent wound is a principle of treatment, its extent is still a matter of opinion.

The treatment of open fractures can be conveniently brought under two headings for the purposes of discussion.

I. The treatment of an already infected open fracture.

2. The treatment of an open fracture before infection has occurred,

covering roughly three eras which can be called (I) pre-antiseptic, (2) antiseptic, (3) post-antiseptic, of which the first and last have the most in common.

Stress is laid on the word "antiseptic" since the introduction of antiseptics-which as a practical application of the work of Lister and Pasteur initiated such a profound effect on the practice of surgery in general-brought about a curious negation of a principle in the treatment of inflammation which-for a time-came to be regarded not as a vital mechanism of defence, but as a condition demanding vigorous local attack.

Such was the position at the beginning of the last war when wounds-the majority of which were heavily infected-were treated by a, free use of the knife, insertion of tubes, followed by frequent dressings and irrigations with antiseptics, necessitating painful disturbance of the limb daily, twice daily, or even more frequently. The principle described so well by John Hilton in his classic Rest and Pain had been replaced by a ceaseless activity, with the consequence that men died from exhaustion, the result of increasing sepsis, aggravated by frequent painful dressings and inadequate immobilisation; lying in dread of the next dressing, the men neither slept nor ate.

During I9I6 ideas were beginning to change, and in certain hospitals work was being carried out that ultimately had a far-reaching effect on the treatment of infected wounds which, though insufficiently noticed at the time, is more fully appreciated to-day. Certain techniques then initiated, mainly perhaps of historic interest, are with modifications in use to-day.

In a hospital at Compiègne, Alexis Carrel established a routine of treatment based on an extensive debridement, including bone fragments, followed by an intermittent irrigation with Dakin's (hypochlorite) solution with only occasional disturbance of the dressings or limb. The whole routine was carried out with extreme care and close attention to detail and within limits had undoubted success; at the time this was attributed mainly to the proteolytic action of the Dakin's solution, but there is little doubt its success was due to the excision and the comfort of comparatively infrequent and painless dressings with-as a consequence-diminished sepsis.

In Paris a South American surgeon-Chutro-dealing mainly with open infected fractures of the lower limb, was using Carrel's method of irrigation of the wound, but paying greater atterition to the general alignment of the limb, which he gained by powerful traction through a metal stirrup threaded over the os calcis under the tendo Achilles; no splint was applied, but the end of the bed was blocked some ro to I 2 inches.

Next, and, in my opinion, most responsible for demonstrating the importance of immobilisation was Sinclair of the R.A.M.C. While not particularly interested in the wound itself, 
Sinclair was deeply interested in the alignment and immobilisation of the limb, for which he used the Thomas's splint for the upper and lower limbs on a modified Hodgson principle. The degree of perfection of alignment and immobilisation Sinclair obtained by his traction-suspension methods was something new at this period in non-operative treatment of fractures, and these methods are in the main those used to-day. By a minimum of interference with the wound itself, but by perfect immobilisation of the limb with bone fragments in good alignment, Sinclair demonstrated that infected open fractures would clean and repair.

At the end of the last war, therefore, there had been a considerable change in the treatment of infected wounds to that existing at the beginning of it. Certain principles were established, or rather re-established, viz., immobilisation of the limb, alignment of the bone fragments and much less interference with the wound itself; a swing back with differences to the preantiseptic era.

A few years later, about I922, Winnett Orr, of Nebraska, working in a mining district and dealing with a number of cases of open fractures and osteomyelitis, carried actual technique a step further by encasing the limb in plaster after the necessary excision or drainage operation. At the time many regarded this as a revolutionary procedure; it wasn't, but it was a further turn in the wheel of non-interference with the wound, which was now impressed on a wider circle, and for this much credit is due to Winnett Orr. The use of plaster provided, too, an important requirement in treatment by providing warmth to the benefit of the limb in general and the wound in particular, by increasing the flow of tissue fluids.

At the beginning of this war, Trueta with his recent experiences in the Spanish War, advocated - in addition to a wider excision of muscle-a modified plaster technique, in effect a skin plaster moulded to the limb which gives greater security for maintaining the alignment of fractured fragments. While therefore the last war started with surgeons thinking in terms of tubes and dressings with dreams of the ideal antiseptic, this war was started with some at any rate distinctly "plaster conscious."

\section{TREATMENT}

The difference between the non-infected and infected open fracture is a matter perhaps of only an hour or so, but the difference in prognosis is considerable. The time of arrival, however, of civilian casualties or service wounded at a hospital or unit equipped for treatment is primarily a transport problem subservient to the military situation; so far air-raid casualties, at any rate all that could be reached, have been received early.

There are essentials of treatment common to the non-infected and the infected fractures, viz., immobilisation of the part wounded with bone fragments in alignment, after the initial treatment non-interference with the wound, and the administration of sulphanilamide. For early cases must be added (I) the treatment of shock, (2) primary amputation, and (3) the excision of the wound. Treatment can be considered under these headings, but with due appreciation that all these items are interdependent.

\section{Shock.}

Though this is not the place to discuss the treatment of shock it must, of course, in a number of cases take preference over any other treatment. On the other hand, time lost before dealing with an open fracture is increasing the risks of infection; the timing therefore of an operative procedure is of the greatest importance. The details of the operation, too, must be influenced by the general condition of the patient, and for these reasons in my opinion the decision as to when to operate should be the responsibility of the operating surgeon after discussion with those responsible for resuscitation measures. A point to be emphasised is that splinting of an injured limb in itself gives some measure of relief to shock, and should be done at the earliest possible moment.

\section{Primary Amputation.}

While certain factors can be mentioned which may influence the decision to perform a primary amputation the indications, both local and general, must be judged in each case. The difference to the individual between an amputation of the upper and lower limb must at all times be borne in mind; speaking very broadly, it may be said that life might be risked for a hand, but not for a foot. Damage to a limb may be such that repair is impossible; also the 
more extensive the damage the greater the danger to life from infection. The desire, therefore, to avoid amputation, while indeed at most times a primary virtue in a surgeon, must not be overstrained to the prejudice of the wounded. Full nursing facilities and appropriate equipment are necessary for any attempt to save the all but destroyed limb, and if these are not available it may influence the decision in a doubtful case. In these respects those receiving wounded at some outlying point are at a disadvantage compared with those receiving air-raid casualties in a big centre.

Extensive injury to soft tissue with division of large nerves, extensive avulsion of skin may necessitate amputation rather than the extent of the injury to a shaft of bone. By some, open disorganisation of the knee-joint with comminution of the condyles is mentioned as an indication for a primary amputation, but in these days of sulphanilamide and arthrodesis this view may alter. Injury to the main vessels of a limb, while not necessarily demanding amputation may do so, and has to be judged by the condition of the limb and the extent of other injuries, but on the whole gross interference with circulation is probably the most definite indication for a primary amputation.

\section{Excision of Wounds.}

The removal of all lacerated and already dead tissue is an essential part of the treatment of an early wound. In order to minimise shock and further shifting of bone fragments while this is performed, the limb or part should be kept as steady as possible; in the lower limb the insertion of an os calcis pin on which to maintain traction is helpful in this respect. The extent of the excision will be influenced by the general condition of the patient, and as a careful and complete excision is the best guarantee against infection every means must be taken to avoid adding to shock while this is done.

Excision of lacerated skin, subcutaneous tissue, aponeurosis and muscle should be performed well clear, say, one-eighth to one-quarter of an inch, of the injured tissue and without thought of primary closure of the wound-partial or complete. In every case so far as is possible, all foreign bodiès should be removed without spending too long a time or opening up uninjured tissue in search of a small fragment. When this part of the operation has been completed, sulphanilamide - to which further reference will be made-is applied to the whole extent of the wound. It is unwise, in my opinion, to attempt the closure of an open fracture in any case: partial closure of the wound may be done, though to have to undermine skin and subcutaneous tissue to accomplish this is risky, as fresh tissues may be exposed to an infection of unknown type and severity. These statements are made as an expression of personal opinion. From certain clinics in the U.S.A. many successful cases have been reported of complete closure of an open fracture after excision and local administration of sulphanilamide. In particular cases this may be done probably with impunity, but to adopt the idea of closure as a routine would be very dangerous, and in war wounds should be entirely discarded. In some cases to aid partial closure incisions are made in the skin and subcutaneous tissues parallel to the wound, but on the whole when loss of tissue prohibits closure my feeling is that a secondary closure of a clean wound is a far better procedure than the primary closure of a wound potentially infected.

As a general rule it is safer to excise a wound, however small, when bone has been injured. In the open fracture, when the skin has been punctured only by a sharp bone fragment from within, it may be urged that excision is unnecessary. In some cases this may be so, but if there be any doubt excision should be performed, after which it may be tempting to close the wound completely, which, as just stated, is being done in a number of cases; on general principles it is safer-even under the most favourable circumstances-to be content with a partial closure, reserving complete closure for a few particular cases. In gunshot wounds an entrance or exit wound if present may be small, but the degree of intervening laceration cannot be estimated, while soiled material may have been carried to any part of the wound and between bone fragments. In certain situations excision of a wound, e.g. a wound of the buttock involving the ilium, is a formidable procedure, yet it is in such situations where soft tissues are massive that an excision of the whole track is of the greatest importance; buttock wounds are notorious for gas infections and secondary haemorrhages from the gluteal vessels.

To what extent should fragments of bone be excised? To refer again to Carrel and the surgeons working with him, it was their routine-influenced mainly by Leriche-to remove subperiosteally all loose bone fragments and soiled ends of the shaft, though fragments involving the whole circumference of a long bone were as far as possible left. Such a procedure of course 
involves removal of lacerated periosteum, but without the complete "esquillectomy," as it was called, this school insisted, with much justification, that soiled material might be left buried between bone fragments, and, too, the procedure was demonstrated to be a considerable factor in limiting sepsis. The demands of the upper and lower limb must again be borne in mind; a loss of bone substance in the arm is fully justified in order to save it, and if saved a degree of function greater than that of an artificial limb can be guaranteed. In the leg loss of bone substance cannot be extensive without being incompatible with function whatever artificial aid is given. The teaching of extensive removal of bone has not been generally accepted, and more especially in the lower limb a compromise has been adopted. This entails a removal of all small, completely loose fragments of bone, on occasion larger fragments if soiling is severe. This measure has been shown fairly conclusively to suffice with the introduction of sulphanilamide therapy, though generally speaking sulphanilamide should be used as a help to the excision, and not to replace it or reduce its scope.

The extent, however, of a debridement must remain a matter for the judgment in each particular case of the operating surgeon, who will be influenced by the general condition of the patient, the site and extent of the wound and the type and degree of its soiling. An extensive debridement is logical, but not always practical. More recent treatment of war wounds in France and Spain extends the scope of debridement to épluchage which entails excision of the whole of the damaged tissues, however deep or extensive. Every recess is opened up and every corner examined, and no dead material allowed to remain.

Good anatomical knowledge is required to avoid main structures which in itself perhaps implies a criticism that debridement or épluchage however extensive is still incomplete. Still by this method many wounds can be transformed into practically aseptic areas; the wound should not be immediately sutured, but secondary suture may be considered after a lapse of 2 to 3 weeks.

Next comes a more difficult question. Should an excision of the wound be performed in an already infected open fracture? This brings other questions in its train, such as: "Is there a time-limit for the performance of an excision?" and "How is it known that a wound is already infected?"

The rate of infection of a wound, or rather the time in which the signs of infection will be manifest, varies with the type of wound. The open lacerations with destruction of bone and soft tissues are, of course, more exposed to soiling, and consequently to infection; other wounds vary in proportion to the degree of laceration and exposure. As a rough estimate it may be said that an open lacerated wound is likely to have become infected in about 7 or 8 hours when signs of infection may be already apparent. In other cases there may be no obvious sign of infection up to or after 24 or 36 hours, especially in the more closed wounds, but this does not mean necessarily that an infection is not present, and may still develop perhaps àcutely. While therefore it must be the aim to treat wounds as early within the 7 - or 8-hour limit as possible, excision is not excluded at a later stage provided there are no signs of infection present, but if there are, in my opinion-which must be taken as a personal one-excision in the first instance is a wrong proceeding. The presence of lymphangitis, a blush or oedema of the skin surrounding the wound, an undue dryness of the wounded tissues, or alternatively a thin serous discharge already perhaps offensive, are some local contra-indications to excision in the ordinary case which may be reinforced by systemic disturbance. On the other hand, any indications of the presence of a gas infection demands imperatively an immediate opening of the wound. Intravenous administration of sulphanilamide alone is relied on by some for the treatment of gas infections. There is, however, still uncertainty whether this is effective in all types of gas infection, and for this reason local measures should not be excluded.

It seems doubtful whether sulphanilamide is taken into the bloodstream through infected tissues, but after its administration by mouth, or intravenously, provided sepsis is being controlled, necessary attention to the wound or bone fragments may be justifiable. For the time being perhaps it will be safer to maintain that the only immediate interference with an open infected fracture should be to insure that drainage is established if the wound itself is insufficient for this, followed by immobilisation of the limb and administration of sulphanilamide.

\section{Sulphanilamide.}

When excision of the wound has been completed theexposed tissuesare insufflated thoroughly with sulphanilamide powder, or introduced in the form of an emulsion with glycerine; this is a 
question of personal preference, and is immaterial provided the whole area of the wound is covered. The concentration from this local application is probably sufficient to cover the postanaesthetic period, while the question of continuing sulphanilamide by mouth as a possible prophylactic measure is advocated by some as a routine, by others in particular cases only. After-treatment in this respect will be influenced by the extent of the wound or wounds, the degree of soiling, and the satisfaction felt with the completeness of the excision.

To the wound itself not more than the one original application should be made, since repeated applications are stated to retard the growth of granulation tissue.

\section{Dressing.}

The type of dressing applied to the wound will vary much with individual ideas, perhaps fads, which may include a preference for no dressing at all when plaster is being applied. My own preference is that a wound should be covered with a gauze dressing after a generous application of vaseline, flavine-vaseline, or other substance which avoids sticking of the dressing to the wound and undue dryness of the surrounding skin. Packing of a wound in my opinion is unnecessary, and in the event of the patient having to be transported potentially dangerous.

\section{Alignment.}

It may be taken as axiomatic that the proper circulation of a limb and hence its drainage is dependent on its length and alignment. If, for example, the fragments of a fractured femur are overlapping with consequent crumpling of soft tissues there is an impediment to the general circulation of the limb, prejudicial to resistance to infection and repair; overlapping or angulation of tibial or radial fragments will act in the same way. It is therefore a primary measure of treatment to obtain at any rate a good general alignment of bone fragments.

Alignment of fragments may be gained by immediate reduction or gradual reduction by traction. In many open fractures immediate reduction is more easily obtained at the initial operation because of the wound when manipulation of tibial, radial, ulnar fragments, or of the small bones, may be performed by means of bone-holding forceps. In those cases, e.g. an open fracture of the femur or lower end of the humerus or indeed in other fractures, when considerable enlargement of the eriginal wound is required for the purpose of manipulation by this means, reduction by traction is a safer procedure. This statement applies to open fractures only, and not to closed fractures.

In some of the fracture services in U.S.A., where cases of injury are received at a very early stage, an accurate reduction of fragments is obtained and held by internal splinting. To my mind there is no question about the advantages of an immediate and accurate reduction of bone; the only point is the safety of such a procedure in an open fracture, which again must be judged in each individual case by the degree of injury and soiling.

\section{Immobilisation.}

As already mentioned, the war was started with a general trend towards a closed method of treating wounds by encasing the whole area in plaster. This treatment is an excellent one, as it provides warmth, immobilisation, and prevents interference with or repeated inspection of the wound. There must be, however, reservations and strict ones on the use of this method of which the main, in my opinion, are the question of transport and the necessity for traction. If casualties can be kept under observation at the hospital where the primary operation has been performed, or if in the event of evacuation the journey is reasonably short, by all means use plaster if suitable to the case, but if early evacuation is necessary with the period of transport and eventual disposal uncertain, closure in plaster becomes risky. When wounded Spanish refugees who had been treated in this manner arrived in France, it is stated the condition of the wounds in many cases was such as to demand amputation.

Fixed traction by means of pins embedded in plaster can be effective-at a later stage of treatment it may be useful for prolonged transport-but in the presence of comminution of long bones, sagging of the fragments is apt to occur. With these reservations, however, the method can be used for almost all open fractures. When traction is required the Thomas's splint with the various methods of using it is a good standby, and probably still the best splint for transport purposes. For transport it is used as a Thomas splint with fixed traction, for continued traction more usually as a support for the limb while direct traction is applied to it. To avoid the risk 
of sagging of bone fragments, in the use of a Thomas or Braun's splint the under straps should be reinforced or replaced by plaster. Braun's splint has many advantages for the lower limb in comfort and facilities for nursing, but should not be used for transport.

So far as is possible for the purposes of splinting, the limb should be in a position of rest, in itself an aid to the relaxation of muscles and maintenance of the general alignment. A faulty alignment, however, cannot be corrected by the position of a limb; splinting-and if necessary traction--.with the limb in a rest position should be used to maintain an alignment already gained. The rest position of the hand or forearm is with the fingers slightly flexed, the hand in semi-pronation and the elbow flexed to a right angle: it is in this position that rotatory displacements of fractured fragments are corrected. The position of choice for the arm is, in my opinion, by the side when the weight of the limb below the site of fractures is itself a means of traction. If for open fractures in the region of the shoulder joint a thoraco-brachial plaster is applied the arm should be abducted some $40-50^{\circ}$ from the side in order to permit of comfortable application of the plaster.

The position of rest in the lower limb is with an appreciable degree of external rotation, the knee slightly flexed with the foot, neither inverted nor everted, supported at a right angle to the leg.

Splinting of a limb is largely an individual affair demanding a desire to splint and ingenuity in its performance. If the main essentials are complied with, viz., immobilisation, alignment, comfort, the type of splint or particular method of applying it is relatively unimportant; the test is whether it is effective. Constant after-care with attention to detail is all important, and those with this interest in the treatment of fractures, prepared to spend time over their cases, will be repaid by a good measure of success.

The other means of immobilisation of bone fragments as apart from the limb itself is by means of an internal splint of which the plate is far the most effective. When Arbuthnot Lane originated the operation of plating fractures he converted deliberately a closed into an open fracture for the purpose of an accurate reduction of the bone fragments and their fixation. Lane was then of the opinion that his method should never be used for an already open fracture because of the potential infection of unknown severity. At the beginning of the last war fixation of bone fragments in war wounds was attempted, fortunately in only a few cases, but with mainly disastrous results, and in this country it has not been used so far as can be ascertained for the ordinary open fracture of civil life. It is of great interest therefore that as already mentioned in certain fracture services in U.S.A. plating is being used as a method of fixation of the bone fragments of an open fracture. When union of the fracture is complete the plate is removed. As already stated, there is no question about the advantages of an immediate and accurate reduction with the added certainty of fixation afforded by a plate; judgment must rest on the safety of such a procedure. It would be running quite unjustifiable risks to insert a plate in open fractures caused by gunshot, or in any case in which foreign bodies of any type had been driven into the wound and their complete clearance was a matter of uncertainty. The method has been mentioned, not because it should ever be used for war casualties-indeed only under the most favourable conditions should it be attempted in civil casualties-but as an illustration of the cycle in which the various techniques of treatment tend to lapse and reappear.

\section{Non-Interference.}

Attention has already been drawn to the striking contrast between the recognised aftertreatment of wounds at the beginning of the last war, with its frequent dressings and disturbances, and the closed treatment of wounds now largely adopted. It is not easy apparently for everyone to leave a wound alone; a certain urge to "look at" and interfere does persist with a desire, perhaps an increasing one, for a window to be made in a plaster for the purpose of what the French call "surveillance." Probably there is but little harm in an occasional inspection of a wound; on the other hand, is there any benefit beyond a certain moral satisfaction for the inspector? "The removal of dressings, the exposure and dressing of the wound, the general disturbance of an injured person are details which - in an extensive wound or multiple woundsamount to trauma. It is not difficult to realise therefore, that repeated dressings of a wound or wounds apart from the pain may prove a menace rather than a benefit by promoting a risk of introducing an infection, or of aggravating an already existing one. It is important to be clear on the main objects of after-care in these cases. If a wound is infected and suppurating it is essential that drainage should be adequate; provided this is so, pus on the surface of a wound 
is of no consequence, and can be left to discharge itself into dressings or plaster for as long a period as is required, or as is compatible with the general hygiene of a ward! If, however, there is evidence of pus under tension which remains unrelieved, action should be taken.

In my opinion there are three indications for the inspection of a wound or part wounded, though of course others may arise:

(I) Pain; more than the general discomfort expected after a severe injury. An injured limb should not be unduly painful after it has been splinted.

(2) Signs of interference with circulation, e.g. oedema, which persists, discoloration, coldness of the skin, etc.

(3) $A$ sudden or persistent systemic disturbance; a sudden sharp rise in the pulse rate may be the first indication of a gas infection.

The case of open fracture that has been received early, the wound excised, the limb comfortably splinted in good alignment, and is obviously making uneventful progress may well be left to itself for at any rate some weeks. The same remarks apply to the after-treatment of a case in which sepsis is present. At all times, though treatment of each case must be strictly on its individual merits; it is so easy to ride a particular hobby-horse too far when non-interference as a policy of treatment may be overdone as much as the policy of trying to save already destroyed limbs. If, when a case is first received, it is considered the condition is such that in the aftertreatment easy access to the wound is going to be important, splinting should be arranged so that it complies with the essentials-but at the same time permits of easy access to the wound. In such a case you may find that a plaster splint with a window is not altogether satisfactory, and that splinting on a Thomas or Braun's splint with an under support of plaster and traction, if required, is preferable in the early stages.

Finally, all cases of open fracture (or closed) should be protected until it is certain that union is firm. Even in those cases without any apparent sepsis and in good alignment, union is slow compared with the closed fracture-mainly because of the delay in formation of ensheathing callus-and perhaps particularly slow in those areas of bone uncovered by muscle. (It is stated that at the present time there is a general tendency throughout the country for union in cases of fracture, open or closed, to be delayed.) In all cases there is the need for treatment under conditions which permit of a generous supply of food, light and air, combined with an activity of the body so far as is compatible with the necessary immobilisation of part of it; nor should the activity of mind be neglected. Rehabilitation of the body-with an avoidance of boredom -is an important factor in maintaining the morale necessary to cope with weeks ro maybe months of disability.

\title{
THE TREATMENT OF OSTEOARTHRITIS BY INTRA-ARTICULAR INJECTION OF LIPIODOL AND GOMENOL'
}

\author{
By ERNEST FLETCHER, M.A., M.B., M.R.C.P. \\ (Physician to British Red Cross Clinic for Rheumatism, Assistant Physician \\ to the Royal Free Hospital)
}

Osteoarthritis is not a lethal disease, but its impact on the efforts of a nation at war is particularly severe because of the prolonged period of disability which it causes.

There are at present no reliable figures to indicate the incidence or average period of disability in cases of osteoarthritis. Its incidence has been shown to be heavy, for in the Ministry of Health report of $\mathrm{I924}$ it was estimated that there were 372,000 cases of rheumatism under national insurance, and in I928 Sir Walter Kinnear estimated the annual cost of rheumatism at more than $£ 5,000,000$. If wages lost through disability were added, he estimated the total cost at $f \mathrm{I} 7,000,000$. Housewives who represent such a large proportion of the population are not as a rule included in national health insurance figures.

We must now consider the proportion of rheumatism which can properly be called osteoarthritis. In a recent series (not yet published) of I,000 cases of chronic rheumatism, the following figures represented this group. 